\title{
Investigation of Borrelia burgdorferi antibodies in patients with multiple sclerosis
}

\author{
Nilay IIdız', İbrahim Halil Özerol², A. Cemal Özcan³ ${ }^{3}$ Hamit Çelik ${ }^{4}$ \\ ${ }^{1}$ Assistant Professor and Head, Department of Pharmaceutical Microbiology, Faculty of Pharmacy, Erciyes University, \\ Kayseri/Turkey, ${ }^{2}$ Professor and Head, Department of Medical Microbiology, Faculty of Medicine, Inonu University, \\ Malatya/Turkey, ${ }^{3}$ Professor and Head, Department of Neurology, Faculty of Medicine, Inonu University, Malatya/Turkey, \\ ${ }^{4}$ Specialist Doctor, Department of Neurology, Buhara Hospital, Erzurum/Turkey
}

Background: Lyme is a disease that is non-compulsory in our country and whose seroprevalence data is less studied. Aims and Objective: Recent studies have shown that bacterial and viral infections are risk factor for various neurodegenerative diseases such as multiple sclerosis and Alzheimer's disease. Herein, we aim to determine the seroprevalence of Lyme in multiple sclerosis (MS) patients. For this purpose, $100 \mathrm{MS}$ patient's serums were investigated for Borrelia burgdorferi IgM and IgG positivity. Materials and Methods: The results identified with ELISA as positive antibody was confirmed by Western Blot (WB) test. The correlation between ages, gender, occupation, tick history, existence of erythema chronicum migrans (ECM), antibody positivity, pain, year with MS results were investigated using Kolmogorov-Smirnow and Kruskal-Wallis statistical test. Results: $B$. burgdorferi IgM and IgG antibodies were positive in $8 \%$ patients when using ELISA method, but that were found to be $2 \%$ by WB. ELISA IgM antibody test gave a 5 negative result in WB. These results were considered false positive in the ELISA test. So, altogether 5 patients were positive by WB method. None of syphilis positive samples detected that $B$. burgdorferi positive serum. A significant difference between the parameters in terms of IgM positivity was not detected $(p>0.05)$. B. burgdorferi IgG antibodies were found significant differences between the MS disease duration ( $p=0.03$ ). MS in the group of less than 10 years had higher titers of IgG antibodies to $B$. burgdorferi. Conclusion: Although a small number of patients with MS is positive with Lyme antibodies. Lyme disease is a treatable.Also, If the patient is MS, clinician should be considered Lyme in the differential diagnosis. This is the first study that the correlation between Lyme and MS from Turkey.

Key words: B. burgdorferi; Multiple sclerosis; ELISA; Western blot; Lyme disease

\section{INTRODUCTION}

Borrelia burgdorferi is the first time isolated from the genus Ixodes ticks in 1977 and the bacteria was first detected in the 1990s in our country. ${ }^{1,2,3} \mathrm{It}$ is transmitted to people by the bite of ticks infected with B. burgdorferi and causes Lyme disease. Lyme neuroboreliosis is nervous system diseases that also can be seen in some of Lyme patients. ${ }^{4}$

Multiple sclerosis (MS) is an autoimmune disease that causes inflammation, plaques and damage to the myelin sheath of neurons in the brain and spinal cord. Insufficient production of myelin or myelin destruction by the immune system is thought to be the cause. Genetic and environmental factors such as infections has been suggested as a cause of myelin damage. MS is usually diagnosed based on signs and symptoms and supporting medical tests results. Lyme neuroborreliosis seen in neurological inflammatory reactions, can be seen in patients with MS and demonstrates similar pathological processes. ${ }^{5,6,7}$ Lyme disease is a result of mimicking neurological symptoms of MS, therefore; these correlation can be misdiagnosed.

There has been a parallelism between the prevalence of $B$. burgdorferi antibodies in MS worldwide where B. burgdorferi as endemic, especially in America and Europe., 
B. burgdorferi-infected tick bite history in people are reported who are also diagnosed with MS. In some of the B. burgdorferi infection, it was observed that peaked at similar times MS seasonally. ${ }^{8}$ In experimental studies, cerebrospinal fluid from MS patients were given to rhesus monkeys and found that a few months after the formation of B. burgdorferi antibodies in these animals. ${ }^{9}$ Also of minocycline, an antibiotic that is effective against B. burgdorferi, has been shown to relieve symptoms in some patients with MS. ${ }^{8}$

Glycolipid antigen BbGL-2 of B. burgdorferi and the myelin major components glycolipid galaktocerobrosides $(\mathrm{GalC})$ are shown structural similarities. According to the molecular similarity theory, as a result of immune response some antibodies are produced. Then they attack to the structural similar myelin sheath antigens and as a result of these condition MS symptoms gets started. In principle autoimmune antibodies (anti- GalC) attack the myelin sheath antigen that causes demyelinating. ${ }^{10}$

Lyme disease is commonly observed in Europe and the United States (US) and include mandatory notification diseases. In our country, there is small number of case reports and serological studies. These are not included in mandatory notification diseases. In this study, B. burgdorferi antibody are investigated in patients with MS.

\section{MATERIALS AND METHODS}

MS patients $(n=100)$ were studied at outpatient visits to the Department of Neurology clinics of Turgut Ozal Medical Center, Inonu University Hospital, Malatya, Turkey for 14-month period between June 2011 and August 2012. The subjects must be under the age of 18 years as inclusion criteria for the study and receiving a definite diagnosis of MS criteria. Patient consent forms and questionnaires were filled out by patients. Retrospective evaluation was performed according to the information of the patients.

ELISA and immunoblot were determined by commercially available kits. IgGandIgM antibodies were studied with Anti-Borrelia plus VIsE ELISA IgG and anti-Borrelia ELISA IgMkits, and immunoblotmethod were studied with Anti-Borrelia EUROLINE-RN-AT (Euroimmun, MedizinischeLabordiagnostika AG).

As a result, samples found positive were also examined for syphilis in order to eliminate false positivity. Firstly, anti-syphilis test (Venereal Disease Research Laboratory, Laboquick, Turkey) were used. The suspicious results were reassessed by using another syphilis detection method, the TPHA (T. pallidium Hemagglutination test, Omega Diagnostic, UK). No positive syphilis results were detected.

\section{RESULTS}

In this study, a total of 100 MS patients were included where 74 were women and 26 were men. The mean age of patients were found 34 and the age varies between of $18-59$ years. Non-working population were identified as $80 \%$ of patients. Only 2 patients gave a clear answer on that exact contact with ticks. 95 of the patients said they have no contact with ticks, 3 patient said they don't remember. Presence of erythema chronicum migrans like-lesion was $8 \%$. It was determined that the joint pain is present in $64 \%$ of patients.

Statistical analysis were performed using SPSS v.16.0 program. As a result of the examination of the normality assumption of the distribution of the findings according to age and gender, the findings of our study showed normal distribution according to the KolmogorovSmirnow test. Also, there was no significant difference between the groups according to the Kruskal-Wallis test. Patients terms of elapsed time from diagnosis of the disease so far, and they are divided into two groups with $0-10$ years and over 11 years. Independent variables were age, sex, occupational group, tick history, ECM existence, pain, and MS years. General characteristics of these variables are shown in Table 1. MS compared to the variable $B$. burgdorferi positive findings concerning the $\mathrm{IgM}$ and $\mathrm{IgG}$ are shown in Table 2.

Groups according to the normal distribution was detected and Kruskal-Wallis test. Also, the results analysed using Kolmogorov-Smirnow test no significant differences ( $>$ > 0.05) examined by the normality of the distribution between the groups age and gender.

\begin{tabular}{llcc} 
Table1: Independent variables & & \\
\hline Variables & Options & $\mathbf{n}^{*}$ & $\%$ \\
\hline Age (years) & $18-28$ & 26 & 11 \\
& $29-38$ & 36 & 36 \\
& $39-48$ & 27 & 27 \\
Sex & 49 years and over & 11 & 11 \\
& Female & 74 & 74 \\
Vocation & Male & 26 & 26 \\
& Jobless & 80 & 80 \\
Tick story & Employee & 13 & 13 \\
& Student & 7 & 7 \\
\multirow{4}{*}{ Erythema chronicum } & None & 95 & 95 \\
migrans similar lesion & Not remember & 3 & 3 \\
Joint pain & Remember & 2 & 2 \\
& None & 92 & 92 \\
& Present & 8 & 8 \\
MS year & Present & 64 & 64 \\
& None & 23 & 23 \\
& Unclear & 13 & 13 \\
*: indicates the number of participants & 92 & 92 \\
& 0-10 years & 8 & 8 \\
\hline
\end{tabular}

Asian Journal of Medical Sciences | Mar-Apr 2020 | Vol 11 | Issue 2 


\begin{tabular}{|c|c|c|c|}
\hline Variables & Diagnosis year of MS & $\mathbf{n}$ & $p$ \\
\hline B.burgdorferi IgM & $\begin{array}{l}0-10 \text { years } \\
11 \text { years and over }\end{array}$ & $\begin{array}{c}92 \\
8\end{array}$ & 0.219 \\
\hline B.burgdorferi lgG & $\begin{array}{l}0-10 \text { years } \\
11 \text { years and over }\end{array}$ & $\begin{array}{c}92 \\
8\end{array}$ & 0.03 \\
\hline
\end{tabular}

Borrelia burgdorferi $\operatorname{Ig} G$ variable based on the period of 0-10 years and 11 years and over found significant differences between the MS patients. The p-value was calculated as 0.03 . Considering the mean of $0-10$ years of in patients with MS appear to be higher value of B. burgdorferi $\operatorname{IgG}$ antibodies. There were no significant differences between the results obtained at all $(p>0.05)$ when compared with ELISA and WB method results.

The present study confirmed the positive ELISA test result with WB. 5 WB method of false-positive IgM antibodies were detected. But compared with the state variables of the study it did not cause a statistically significant difference $(\mathrm{p}>0.05)$.

\section{DISCUSSION}

Lyme disease prevalence is not clear in our country because of it can be confused with other diseases and it isn't take place in the list of notifiable diseases. In our country, a limited number of study made Lyme disease agent (B. burgdorferi) seropositivity in individuals with risk group (B. burgdorferi antibodies were in the village living or livestock dealing) by 6\% in Ankara, 6.6\% in Trabzon, 22.1-35.9\% in Antalya. Also it has been reported as $18.9 \%$ in Denizli and $7.8 \%$ in $\mathrm{Izmir}^{10}$. In healthy individuals, patients with a history of tick bites shows $17 \%$ seropositivity. In non-bite history, $2 \%$ seropositivity was found in a study conducted in Isparta, ${ }^{11}$ However, in these studies, validation testing has not been done.

Chmielewska-Badora et al investigated some disorders. 149 of them were determined as neurological diseases. In these people, 26 patients were MS and also 10 of these 26 MS patients were determined as B. burgdorferi seropositive $(p=0.042)^{5}$. Karussis et al in a single case study examined the treatment of a patient with severe MS for five years. At the end of the fifth year he came unable to work. The analysis was performed towards various viral agents and B. burgdorferi. These tests revealed that WB tests results of the patient have been found to he was neuroborreliosis and one year of treatment resulted 59\% improved in patient function. ${ }^{12}$ This is the case in patients with multiple sclerosis if Lyme disease should not be ignored and treated patients showed a significant improvement in providing evidence.
Boronava et al reported that in endemic area differential diagnosis of MS and Lyme disease is an important. ${ }^{1}$ In contrast, another study in 55 patients with a definite diagnosis of MS- Lyme researched and only 3 were detected seropositive in patients and differential diagnosis of unknown etiology diseases such as MS, Lyme disease has been concluded that it is important to bear in mind. ${ }^{7}$ There are no studies in the literature in our country in this field. This is the first study that unclose a correlation between Lyme and MS from Turkey republic.

In our study, more than 10 years of ECM lesions in patients with MS were found to be significantly related with Borrelia IgG levels $(p<0.05)$. The results that are unrecognized or untreated Lyme disease even in cases of Lyme disease MS is to mimic nature shows.

In people with Lyme disease months or years after chronic encephalopathy, polyneuropathy, or may occur less frequently leukoencephalopathy. These chronic neurological abnormalities usually resolve with antibiotic treatmen $t^{13}$ so early or late in terms of disease can be treated and it is important to distinguish the difference from MS.

Mansouri and friends said that MS in women compared to men in a study investigating the risk values encountered more frequently in people with MS 3/1 percent and the average age was found to be $32 .{ }^{14}$ In a similar study we found that $76 \%$ of female patients with MS, it is observed that the average age of 34 .

As a result, Lyme disease can cause lesser extent mistake in the diagnosis of neurological symptoms resemble symptoms of MS. Therefore, in areas with a high population of ticks Ixodes genus, the prevalence of B. burgdorferi reservoir animals should be determined. In patients with MS, bearing in mind in the differential diagnosis with Lyme disease, we propose the investigation of B. burgdorferi antibodies.

\section{ACKNOWLEDGEMENTS}

\section{Funding}

This study was produced from the doctoral thesis of Nilay (Gucluer) Ildiz. We gratefully acknowledge to Inonu University for financial support for this study with projectnumber: 2011/169. We also acknowledge the physicians of the MS Clinic of Turgut Ozal Medical Center for their help in this study.

\section{REFERENCES}

1. Baranova NS, Spirin NN, Fadeeva OA, Shipova EG, Stepanov IO. Lyme disease in patients with multiple sclerosis: clinical, diagnostic and therapeutic features. Zh Nevrol Psikhiatrlm S S 
Korsakova 2012; 112: 64-68.

2. Blewett MM. Hypothesized role of galactocerebroside and NKT cells in the etiology of multiple sclerosis. Med hypotheses 2008;70: 826-830.

3. Burgdorfer W, Barbour AG, Hayes SF, Benach JL, Grunwaldt E and Davis JP. Lyme disease-a tick-borne spirochetosis? Science 1982; 216: 1317-1319.

4. Bulut C, Koçak TZ, Altun Ş, Altınel E, Kınıklı S and Pekcan Demiröz A. An overlooked disease of tick bites: Lyme disease. Mikrobiyol Bul 2009; 43:487-492.

5. Chmielewska-Badora J, Cisak E and Dutkiewicz J. Lyme borreliosis and multiple sclerosis: any connection? A sero epidemic study. Ann Agric Environ Med 2000;7 (2):141-143.

6. Fritzsche M. Chronic Lyme borreliosis at the root of multiple sclerosis-is a cure with antibiotics attainable?.Medical Hypotheses 2005; 64: 438-448.

7. Garcia-Monco JC, Miro-Jornet J and Fernandez Villar B. Multiple sclerosis or lyme disease? a diagnostic problem of exclusion. Med Clin 1990; 94: 685-688.

8. Fritzsche M. Chronic Lyme borreliosis at the root of multiple sclerosis-is a cure with antibiotics attainable? Medical Hypotheses 2005; 64: 438-448.

9. Adams DK, Blacklock WS and Cluskiie JAW. Spirochaetes in ventricular fluid of monkeys inoculated from cases of disseminated sclerosis. J Pathol Bacteriol 1925; 28:117-118.

10. Uyanık M. H, Yazgı $H$ and Ayyıldı A. Seropositivity Of Lyme Disease In Erzurum Province, Turkey. Turkish Journal of Infection 2009; 23: 69-72.

11. Güneş T, Poyraz Ö, Kaya S, Gençer L and AlimA. Sivas Yöresinde Borrelia Burgdorferi Vektörlerinin Ve Lyme Seropozitifliğinin Araştırılması, Mikrobiyol Bült 2005; 39: 503-508.

12. Karussis $\mathrm{D}$, Weiner $\mathrm{HL}$ and Abramsky $\mathrm{O}$. Multiple sclerosis vs Lyme disease: a case presentation to a discussant and review of the literature. Mult Scler 1999; 5: 395-402.

13. Logigian EL, Kaplan RF and Steere AC. Chronic neurologic manifestations of Lyme disease. N Engl J Med 1990; 323:1438-1444.

14. Mansouri B, Mansouri B, Asadollahi S, Heidari K, Fakhri M, Assarzadegan $F$, et al. Risk factors for increased multiple sclerosis susceptibility in the Iranian population. J Clin Neurosci 2014; doi: 10.1016/j.jocn.2014.04.020.

Authors Contribution:

$\mathrm{NI}$ and IHO- Designed the project. made contributions on experimental section. wrote the manuscript; NI- studied all the experiments; ACO and HC- Helped with patient selection, clinical study.

Work attributed to:

Department of Pharmaceutical Microbiology, Faculty of Pharmacy, Erciyes University, 38039 Kayseri/Turkey.

Orcid ID:

Dr. Nilay Ildız - (D) https://orcid.org/0000-0002-3799-856X

Source of Support: Nil, Conflict of Interest: None declared. 